\title{
Does dynapenic abdominal obesity accelerate the decline in physical performance in older adults?
}

Roberta de Oliveira Máximo

Federal University of Sao Carlos

Dayane Capra de Oliveira

Federal University of Sao Carlos

Paula Camila Ramirez

Federal University of Sao Carlos

Mariane Marques Luiz

Federal University of Sao Carlos

Aline Fernanda de Souza

Federal University of Sao Carlos

Maicon Luís Bicigo Delinocente

Federal University of Sao Carlos

Andrew Steptoe

University College London

Cesar de Oliveira

University College London

Tiago da Silva Alexandre ( $\square$ tsfisioalex@gmail.com )

Federal University of Sao Carlos https://orcid.org/0000-0003-3791-9793

\section{Research}

Keywords: waist circumference, grip strength, SPPB, physical performance, trajectories, older adults, ELSA

Posted Date: April 28th, 2020

DOl: https://doi.org/10.21203/rs.3.rs-23563/v1

License: (c) (i) This work is licensed under a Creative Commons Attribution 4.0 International License. Read Full License 


\section{Abstract \\ Background}

There are few epidemiological evidences of sex differences in the association between dynapenic abdominal obesity and the decline in physical performance among older adults.

\section{Objectives}

To investigate whether the decline in physical performance is worse in individuals with dynapenic abdominal obesity and whether there are sexes differences in this association.

\section{Methods}

A longitudinal analysis was conducted with 3,881 participants of the English Longitudinal Study of Ageing aged 60 years or older in an eight-year follow-up period. The outcome was physical performance evaluated using the Short Physical Performance Battery (SPPB). Abdominal obesity was determined based on waist circumference ( $>102 \mathrm{~cm}$ for men and $>88 \mathrm{~cm}$ for women). Dynapenia was determined based on grip strength ( $<26 \mathrm{~kg}$ for men $<16 \mathrm{~kg}$ for women). The sample was divided into four different groups: nondynapenic/non-abdominal obese (ND/NAO); non-dynapenic/abdominal obese (ND/AO); dynapenic/nonabdominal obese (D/NAO); and dynapenic/abdominal obese (D/AO). Changes in SPPB performance levels in these groups, stratified by sex, were analyzed using generalized linear mixed models adjusted by sociodemographic, behavioral and clinical characteristics.

\section{Results}

At baseline, women with D/AO had the worst performance on the SPPB among the groups analyzed (-1.557 points; $95 \% \mathrm{Cl}$ - -1.915 to $-1.199 ; \mathrm{p}<0.001)$, and men with $\mathrm{D} / \mathrm{AO}$ had a worse performance on the SPPB compared to those in the ND/NAO and ND/AO groups (-1.179 points; $95 \% \mathrm{Cl}-1.639$ to $-0.717 ; p<0.001)$. Over the eight-year follow-up, men with $\mathrm{D} / \mathrm{AO}$ had a faster decline in performance on the SPPB compared to those in the ND/NAO group $(-0.106$ points per year; $95 \% \mathrm{Cl}:-0.208$ to $-0.004 ; p<0.05)$.

\section{Conclusion}

Dynapenic abdominal obesity accelerates the decline in physical performance in men but not women.

\section{Introduction}

The decline in physical performance is commonly the first indicator of compromised functioning in older adults $^{1,2}$ and is considered a preclinical transition phase towards disability ${ }^{3-9}$. Screening for this decline 
has been widely defended in the clinical geriatric setting ${ }^{10}$, as it predisposes older adults to a greater risk of negative outcomes ${ }^{1,11}$. The age-related reduction in muscle strength and buildup of fat (dynapenia and obesity, respectively) are known to be independently associated with the decline in physical performance. However, conflicting findings are reported regarding the association between dynapenic obesity and this outcome.

Bouchard and Janssen analyzed 2,039 men and women aged 55 years or older in a cross-sectional study and found that dynapenic obesity (determined as the highest tertile of body fat mass and lowest tertile of knee extensor strength) was associated with self-reported poorer physical function ${ }^{12}$. Yang and collaborators found similar results in a cross-sectional study involving 616 Chinese men and women age 60 years or older, in which dynapenic obesity was defined as a body mass index (BMI) equal to or higher than $25 \mathrm{~kg} / \mathrm{m}^{2}$ and the lowest tertile of grip strength ${ }^{13}$. In contrast, Batsis and collaborators conducted a longitudinal study with a four-year follow-up period involving 2,025 men and women aged 60 years or older and found no evidence of a worse decline in physical performance among individuals with dynapenic obesity, which was defined as a BMI equal to or higher than $30 \mathrm{~kg} / \mathrm{m}^{2}$ and the lowest tertile of knee extensor strength.

The buildup of abdominal fat with age, independently of the BMI, has been associated with high levels of adipocytokines and greater insulin resistance, mediating the decline in neuromuscular strength. Furthermore, this buildup of abdominal fat occurs earlier in men and later and subtler in women, generally after menopause ${ }^{14-16}$. Despite having greater muscle mass and strength than women ${ }^{17,18}$, men experience a more accentuated process of dynapenia ${ }^{17,19}$. All these factors indicate that there may be differences in the effect of dynapenic abdominal obesity on the trajectories of the decline in physical performance between the sexes.

Therefore, the aim of the present study was to test two hypotheses: a) the decline in physical performance is worse in individuals with dynapenic abdominal obesity; and b) there are sex differences in the trajectories of this decline.

\section{Methods}

\section{Study population}

The data used in this study came from the English Longitudinal Study of Ageing (ELSA), which is an ongoing panel study involving community-dwelling individuals in England aged 50 years or older. ELSA began in 2002 and the sample was composed of participants of the Health Survey for England, which was a study involving a nationally representative sample using random probability stratified in different stages

${ }^{20}$. After baseline, follow-up interviews in ELSA occurred every two years and health examinations were performed by a nurse every four years. The first health examination occurred in 2004-2005. A detailed description of the study can be found in a previous publication ${ }^{21}$. 
The sample of the present study comprised 6,183 individuals aged 60 years or older in 2004, when anthropometric and physical performance data were collected for the first time. Among these individuals, 2,302 were excluded due to missing data on the Short Physical Performance Battery (SPPB), grip strength, waist circumference or other covariates, resulting in a final sample of 3,881 individuals at baseline. These measures were not obtained for individuals who were incapable of 1) performing the walk tests without the use of a gait-assistance device; 2) standing up from a chair a single time without using the arms; 3 ) perform the standing balance tests; 4) performing the grip strength test; or 5) remaining in the standing position for the measurement of waist circumference. The participants were reevaluated after four (2008) and eight (2012) years.

\section{Physical performance}

The SPPB is used to determine the physical performance of older adults through the combined assessment of static balance (feet side by side, semi-tandem and tandem), the 2.4-meter walk test and repeated chair stands ${ }^{22}$. The complete battery ranges from 0 to 12 points, with higher scores denoting a better physical performance ${ }^{5}$. In the present study, the SPPB score was treated as a discrete quantitative variable ${ }^{23}$.

\section{Anthropometric measures and classification of groups}

Grip strength was measured using a manual dynamometer (Smedley, range: 0 to $100 \mathrm{~kg}$ ). During the test, the participant remained standing with the arm alongside the trunk and the elbow flexed at 90 degrees ${ }^{24}$. Three maximum strength tests were performed with a one-minute rest period between readings and the highest value was considered for the analysis. Dynapenia was defined as grip strength $<26 \mathrm{~kg}$ for men and $<16 \mathrm{~kg}$ for women ${ }^{25}$.

Waist circumference was measured using a metric tape at the midpoint between the lowest rib and the upper edge of the iliac crest. The measurement was made twice at the end of the expiratory phase of the respiratory cycle ${ }^{24}$. A third measurement was performed if the difference between the first two measurements was greater than $3 \mathrm{~cm}$. Abdominal obesity was defined as waist circumference $>102 \mathrm{~cm}$ for men and $>88 \mathrm{~cm}$ for women ${ }^{26}$.

Four groups were created considering the absence/presence of abdominal obesity and dynapenia: nondynapenic/non-abdominal obese (ND/NAO); non-dynapenic/abdominal obese (ND/AO); dynapenic/nonabdominal obese (D/NAO); and dynapenic/abdominal obese (D/AO) ${ }^{27}$.

\section{Covariates}

The socioeconomic variables were sex, age group (60 to $69 ; 70$ to $79 ; 80$ years or more), marital status (with or without a conjugal life), level of education ( 0 to $11 ; 12$ to $13 ;>13$ years) and total household wealth (quintiles).

Smoking was determined by asking the participants whether they were non-smokers, ex-smokers or current smokers. Regarding alcohol intake, the participants were classified as non-drinkers or rare drinkers (up to 
once per week), frequent drinkers (two to six times per week) or daily drinkers ${ }^{28}$. Level of physical activity was determined using an instrument validated by the Health Survey for England ${ }^{29}$ that considers the frequency of participation in vigorous, moderate and mild physical activities (more than once per week, once per week, one to three times per months or almost never). Lifestyle was classified as sedentary (no weekly physical activity) or active (mild, moderate or vigorous physical activity at least once per week) 27 .

Health status was ascertained by self-reported doctor diagnosis of diabetes, systemic arterial hypertension, stroke, heart disease, lung disease, cancer, joint disease, osteoporosis and falls in the previous 12 months. Cognitive function was evaluated based on the global score of immediate and delayed recall (range: 0 to 20 words) ${ }^{30}$. Depressive symptoms were determined using the Center for Epidemiologic Studies Depression Scale (CES-D), considering a cutoff of $\geq 4$ points ${ }^{31}$.

The percentage change in weight between waves was analyzed to adjust the models, as weight loss can affect the relation between abdominal obesity and the decline in muscle strength. This variable was categorized as follows: stable weight, weight loss equal to or greater than $5 \%$ and weight gain equal to or greater than $5 \%$ compared to the previous wave $\mathrm{e}^{32}$.

All covariates included in the analyses represent a wide range of risk factors associated with the progression of the decline in physical performance ${ }^{33}$. With the exception of age, all variables were treated as time-varying covariates (whenever a variable changes over time for the subjects) ${ }^{34}$.

\section{Statistical analysis}

Differences in baseline characteristics between (a) included individuals and those excluded due to missing data on the SPPB, grip strength, waist circumference or other covariates and (b) the four groups classified based on the presence/absence abdominal obesity and dynapenia were evaluated using the chi-squared test, analysis of variance (ANOVA) and Tukey's post hoc test. For all analyses, a p-value $<0.05$ was considered indicative of statistical significance.

Generalized linear mixed models stratified by sex were used to estimate the trajectories of physical performance using the XTMIXED procedure in Stata 14 SE (Stata Corp, College Station, TX, USA). These models were chosen because they are more appropriate for unbalanced data from studies with repeated measures and enable the statistical modeling of time-dependent changes in the outcome variable (SPPB) and in the magnitude of associations between variables ${ }^{35,36}$.

The two models (one for each sex) include the interaction between time (in years of follow-up) and the status of abdominal obesity and dynapenia adjusted by the covariables. Univariate analyses were performed to select covariables to incorporate into the final model per sex. Only covariables that presented associations with a $\mathrm{p}$-value $\leq 0.20$ in the univariate analyses were selected for inclusion in the multiple models using the stepwise forward method.

In the final models, the intercept represents the mean estimated difference in performance on the SPPB at baseline between individuals categorized based on the presence/absence of abdominal obesity and 
dynapenia considering the ND/NAO group as the reference category. On the slope, time (in years) indicates whether the performance on the SPPB declines independently of the presence of covariables (that is, whether time per se is the determinant of the decline). The interaction between time and each status of abdominal obesity and dynapenia represents the estimated difference in the annual rate of decline in performance on the SPPB (slope) between each of the three groups (ND/AO, D/NAO and D/AO) and the reference group (ND/NAO), enabling the assessment of the annual rate of change in the SPPB in the four groups. The rates of the decline in performance were compared using the $B$ coefficient and $95 \%$ confidence interval $(\mathrm{Cl})$.

Sensitivity analysis was performed to investigate whether abdominal obesity (yes/no) and dynapenia (yes/no), when analyzed separately, were able to modify the association found between dynapenic abdominal obesity and the decline in physical performance.

In addition, statistics to estimate average population parameters, such as the marginal average, were used from predictions of a previously adjusted model.

\section{Results}

Among the 3,881 participants at baseline, 2,962 and 2,481 were reevaluated at the four-year and eight-year follow-up, respectively. Slightly more than $63 \%$ and the initial sample participated in the three waves and $76 \%$ participated in two waves of the study. The baseline characteristics according to the presence/absence of abdominal obesity and dynapenia, stratified by sex, are displayed in Tables 1 and 2 .

At baseline, the prevalence of D/AO and ND/AO was slightly higher in women than men $(3.7 \%$, [95\% Cl: 3.0 to 4.6 ] versus $2.0 \%$ [95\% Cl: 1.4 to 2.7 ] and $50.3 \%$ [95\% Cl: 48.1 to 52.4 ] versus $42.3 \%$ [95\% $\mathrm{Cl}: 40.1$ to 44.6 ], respectively). No difference in the prevalence of dynapenia alone was found between sexes (3.3\% [95\% Cl: 2.6 to 4.3 ] versus $4.0 \%$ [95\% $\mathrm{Cl}$ : 3.2 to 4.9$]$ ]).

In the analysis comparing the included individuals and those excluded due to missing data, the excluded individuals were mainly women, older, with no conjugal life, had less schooling and income, smoked more and had more cognitive decline, weakness, abdominal obesity, sedentary behavior, depressive symptoms, lung disease, heart disease, diabetes, systemic arterial hypertension, stroke, arthritis and osteoporosis ( $p$ $<0.05$, data not shown).

Table 3 shows the estimated parameters of the generalized linear mixed models adjusted for the change in SPPB as a function of the presence/absence of abdominal obesity and dynapenia per sex in the eight-years of follow up. On the intercept, women with D/AO had the worst performance on the SPPB among the four groups (-1.557 points; $95 \% \mathrm{Cl}:-1.915$ to $-1.199 ; \mathrm{p}<0.001)$. Men with $\mathrm{D} / \mathrm{AO}$ had lower intercept values compared to those in the ND/NAO and ND/AO groups (-1.179 points; $95 \% \mathrm{Cl}:-1.639$ to $-0.717 ; p<0.001)$.

Time was an independent predictor of a poorer performance on the SPPB in both sexes, with a mean annual reduction of 0.140 points per year $(95 \% \mathrm{Cl}:-0.229$ to $-0.051 ; p<0.05)$ in men and 0.172 points per year $(95 \% \mathrm{Cl}:-0.260$ to $-0.085 ; \mathrm{p}<0.001)$ in women. Only men with $\mathrm{D} / \mathrm{AO}$ had a faster rate of decline in the 
SPPB compared to the ND/NAO group. The estimated parameter for the difference in slope between the two groups was -0.106 points per year $(95 \% \mathrm{Cl}:-0.208$ to $-0.004 ; \mathrm{p}<0.05)$ after adjusting for age, income, change in weight, smoking, sedentary lifestyle, diabetes, lung disease, heart disease, stroke, joint disease, depressive symptoms and memory score (Table 3).

In clinical terms, D/AO men showed higher mean SPPB score at baseline compared to D/AO women (9.373 points vs. 8.544 points). However, D/AO men had an average decline of 0.189 points per year for SPPB scores, against decline of 0.067 points per year to D/AO women over the eight-year follow-up (Figures 1 and 2 and Supplementary Table 2).

Compared to the main analysis considering dynapenic abdominal obesity, the results of the sensitivity analysis considering abdominal obesity and dynapenia to be independent conditions showed that although the analyses on the intercept were similar, the analyses on the slope revealed that neither of the two conditions alone was associated with a greater decline in performance on the SPPB (Supplementary Table 1). This highlights the importance of the analytical approach adopted in the present study (considering the influence of combinations of abdominal obesity and dynapenia on the long-term decline in physical performance among older adults).

\section{Discussion}

In this population-based cohort, we demonstrated that older English men with dynapenic abdominal obesity have an accelerated decline in physical performance. Moreover, when analyzing abdominal obesity and dynapenia as independent conditions, neither was associated with an accelerated decline in physical performance in either men or women, which highlights the importance of dynapenic abdominal obesity as a clinical condition.

Previous studies offer divergent findings regarding the association between dynapenic obesity and poorer physical performance in older adults. In a cross-sectional study involving 616 men and women aged 60 years or older, Yang and collaborators ${ }^{13}$ found that individuals with dynapenic obesity had a poorer physical performance than those who did not have obesity or dynapenia. Bouchard and Janssen ${ }^{12}$ report similar results in a cross-sectional study analyzing 2,039 individuals aged 55 years or older. In contrast, Batsis and collaborators conducted a four-year follow-up study involving 2,025 individuals aged 60 years or older and although the authors found that dynapenic obesity was associated with limited physical performance at baseline, this was not confirmed in the longitudinal analyses ${ }^{37}$.

The most likely reason for the differences found between cross-sectional ${ }^{12,13}$ and longitudinal ${ }^{37}$ analyses seems to reside in how obesity is measured. General obesity indicators, such as the BMI used in the longitudinal study conducted by Batsis et al. ${ }^{37}$, may not capture age-related changes in the distribution of body fat. Therefore, waist circumference seems to be more appropriate for this assessment in older adults, despite not being as accurate. 
The sex differences found in the present study may be explained by the different patterns of the decline in muscle strength and the distribution of body fat with aging between men and women. Men exhibit more age-related loss of muscle strength 17,19 and accumulate abdominal fat earlier, with greater intensity and a predisposition towards visceral fat deposition ${ }^{14,38}$. Furthermore, abdominal obesity is associated with a decline in a variety of neural and hormonal trophic signs in the muscles, given the link to chronic inflammation and the reduction in tolerance to glucose ${ }^{39}$. Thus, evidence of the association between abdominal obesity and the exacerbation of the process of dynapenia exclusively in men ${ }^{40}$ lends support to the accelerated decline in physical performance in men with dynapenic abdominal obesity. In contrast, the buildup of central fat arises at an older age and in a subtler manner in women ${ }^{15}$, occurring after menopause and with subcutaneous deposition ${ }^{14}$. Thus, a milder production of inflammatory cytokines due to this alternate fat deposition ${ }^{16}$ may attenuate the relationship between dynapenic abdominal obesity and the decline in physical performance in women over time.

The fact that both men and women with D/AO began the study with worse SPPB scores compared to their counterparts in the ND/NAO groups highlights the importance of dynapenic abdominal obesity as a clinical condition that affects physical performance. However, the lack of an association between D/AO and a poorer performance on the SPPB in women over time may reflect the smaller effect of abdominal fat on the loss of muscle strength, which was milder in women than men with dynapenic abdominal obesity $(p<0.01$, data not shown).

Moreover, the sensitivity analyses showed that by not combining abdominal obesity with dynapenia may lead to neglecting important associations between these conditions and the decline in physical performance, as abdominal obesity and dynapenia alone were not associated with the outcome (Supplementary Table 1). This highlights the importance of the analytical approach adopted in the present study.

This study has several strengths. The major strength is the use of a representative national sample of community-dwelling older adults in England, which enabled us to perform analyses stratified by sex. The use of objective measures of health and physical performance (waist circumference, grip strength and SPPB) is another strong point. Moreover, the analyses involved data from three waves and a long follow-up period, which enabled us to detect changes in physical performance over time. We also considered the influence of the regional redistribution of adipose tissue during the aging process and our models were adjusted by a wide range of important covariates associated with both the exposure and outcome.

The present study has also limitations that need to be considered. First, the losses to follow-up may be a source of bias, although this type of bias is inevitable in longitudinal studies involving community-dwelling older adults. Another potential source of bias relates to the generalization of the data, as the participants excluded from the analytical sample were poorer, had worse memory, a sedentary lifestyle and a greater probability of lung disease and diabetes. Despite the differences between the included and excluded individuals, we were able to observe accelerated decline in the physical performance of men with dynapenic abdominal obesity. The lack of information on diet and the history of obesity (onset and duration) 
constitute another limitation. Finally, waist circumference does not provide a direct estimate of visceral adiposity, as achieved with computed tomography and magnetic resonance. However, it is a very useful screening tool in clinical practice.

\section{Conclusion And Implications}

Dynapenic abdominal obesity accelerates the decline in physical performance only in older men. This finding highlights the clinical importance of including abdominal obesity and dynapenia in the evaluation of the risk of decline in physical performance, especially when these two conditions are found in the same patient.

\section{Declarations}

\section{Ethics Approval and Consent to participate}

ELSA received approval from the National Research Ethics Service (London Multicentre Research Ethics Committee [MREC/01/2/91]) and all participants signed a statement of informed consent.

\section{Consent for publication}

All authors meet the criteria for authorship stated in the Uniform Requirements for Manuscripts Submitted to Biomedical Journals.

\section{Availability of data and material}

Data from the English Longitudinal Study of Ageing (ELSA) are available from the UK Data Service for researchers who meet the criteria for access to confidential data, under conditions of the End User License http://ukdataservice.ac.uk/media/455131/cd137-enduserlicence.pdf. The data can be accessed from: http://discover.ukdataservice.ac.uk/series/?sn=200011. Contact with the UK data service regarding access to the English Longitudinal Study of Ageing can be made through the website http://ukdataservice.ac.uk/help/get-in-touch.aspx, by phone +44 (0)1206 872143 or by email at help@ukdataservice.ac.uk.

\section{Competing Interest}

The authors declare that they have no competing interests"

\section{Funding}

This study received funding from the State of Sao Paulo Research Foundation - FAPESP (grant number: 17/26377-4) and was partially supported by the Coordination for the Improvement of Higher Education Personnel - CAPES (Finance code 001). The National Council of Scientific and Technological Development - CNPq (grant number: 303981/2017-2) and FAPESP (grant number: 18/13917-3) support Tiago da Silva Alexandre. ELSA is funded by ESRC-UK and the National Institute on Aging USA (grants numbers: 
5R01AG017644-16 and 5R01AG017644-18) and by a consortium of governmental departments of the United Kingdom coordinated by the Economic and Social Research Council (ESRC).

\section{Author' Contributions}

Study concept and design: Tiago da Silva Alexandre and Roberta de Oliveira Máximo. Acquisition of data: Tiago da Silva Alexandre, Cesar de Oliveira, and Andrew Steptoe. Data analysis and interpretation: Tiago da Silva Alexandre, Roberta de Oliveira Máximo, and Cesar de Oliveira. Preparation of manuscript: All authors. Revising and Final approval: All authors.

\section{Acknowledgements}

Not applicable

\section{References}

1. Cesari M, Kritchevsky SB, Newman AB, et al. Added Value of Physical Performance Measures in Predicting Adverse Health-Related Events: Results from the Health, Aging and Body Composition Study. J Am Geriatr Soc. 2009;57(2):251-259. doi:10.1111/j.1532-5415.2008.02126.x.Added

2. Cesari M, Kritchevsky SB, Penninx BWHJ, et al. Prognostic value of usual gait speed in well-functioning older people - Results from the health, aging and body composition study. J Am Geriatr Soc. 2005;53(10):1675-1680. doi:10.1111/j.1532-5415.2005.53501.x

3. Rantakokko M, Mänty M, Rantanen T. Mobility Decline in Old Age. Exerc Sport Sci Rev. 2013;41(1):1925. doi:10.1097/JES.0b013e3182556f1e

4. Gill TM, Williams CS, Tinetti ME. Assessing risk for the onset of functional dependence among older adults: the role of physical performance. J Am Geriatr Soc. 1995;43(6):603-609. doi:10.1111/J.15325415.1995.TB07192.X

5. Guralnik JM, Ferrucci L, Simonsick EM, Salive ME, Wallace RB. Lower-Extremity Function in Persons over the Age of 70 Years as a Predictor of Subsequent Disability. N Engl J Med. 1995;332(9):556-562. doi:10.1056/NEJM199503023320902

6. Guralnik JM, Ferrucci L, Pieper CF, et al. Lower Extremity Function and Subsequent Disability: Consistency Across Studies, Predictive Models, and Value of Gait Speed Alone Compared With the Short Physical Performance Battery. Journals Gerontol Ser A Biol Sci Med Sci. 2000;55(4):M221-M231. doi:10.1093/gerona/55.4.M221

7. Guralnik JM, Ferrucci L. Assessing the building blocks of function: Utilizing measures of functional limitation. Am J Prev Med. 2003;25(3 SUPPL. 2):112-121. doi:10.1016/S0749-3797(03)00174-0

8. Hardy SE, Kang Y, Studenski SA, Degenholtz HB. Ability to walk $1 / 4$ mile predicts subsequent disability, mortality, and health care costs. J Gen Intern Med. 2011;26(2):130-135. doi:10.1007/s11606-010-15432

9. Shinkai $S$, Watanabe $S$, Kumagai $S$, et al. Walking speed as a good predictor for the onset of functional dependence in a Japanese rural community population. Age Ageing. 2000;29(5):441-446. 
doi:10.1093/ageing/29.5.441

10. Studenski S, Perera S, Wallace D, et al. Physical performance measures in the clinical setting. J Am Geriatr Soc. 2003;51(3):314-322. doi:10.1046/j.1532-5415.2003.51104.x

11. Montero-Odasso M, Schapira M, Soriano ER, et al. Gait velocity as a single predictor of adverse events in healthy seniors aged 75 years and older. J Gerontol A Biol Sci Med Sci. 2005;60(10):1304-1309. doi:10.1093/gerona/60.10.1304

12. Bouchard DR, Janssen I. Dynapenic-obesity and physical function in older adults. J Gerontol A Biol Sci Med Sci. 2010;65(1):71-77. doi:10.1093/gerona/glp159

13. Yang M, Jiang J, Hao Q, Luo L, Dong B. Dynapenic Obesity and Lower Extremity Function in Elderly Adults. J Am Med Dir Assoc. 2015;16(1):31-36. doi:10.1016/j.jamda.2014.06.019

14. World Health Organization. Physical status: the use and interpretation of anthropometry. Report of a WHO Expert Committee. World Health Organ Tech Rep Ser. 1995;854:1-452. doi:854

15. Sternfeld B, Ngo L, Satariano WA, Tager IB. Associations of body composition with physical performance and self-reported functional limitation in elderly men and women. Am J Epidemiol. 2002;156(2):110-121. doi:10.1093/aje/kwf023

16. Snijder MB, van Dam RM, Visser M, Seidell JC. What aspects of body fat are particularly hazardous and how do we measure them? Int J Epidemiol. 2006;35(1):83-92. doi:10.1093/ije/dyi253

17. Roubenoff R. Origins and clinical relevance of sarcopenia. Can J Appl Physiol. 2001;26(1):78-89. doi:10.1017/CB09781107415324.004

18. Goodpaster BH, Park SW, Harris TB, et al. The Loss of Skeletal Muscle Strength, Mass, and Quality in Older Adults: The Health, Aging and Body Composition Study. Journals Gerontol. 2006;61A, 10(November):1059. doi:10.1093/gerona/61.10.1059

19. Hughes VA, Frontera WR, Wood M, et al. Longitudinal muscle strength changes in older adults: influence of muscle mass, physical activity, and health. J Gerontol A Biol Sci Med Sci. 2001;56(5):B20917. doi:10.1093/gerona/56.5.B209

20. Mindell J, Biddulph JP, Hirani V, et al. Cohort profile: The health survey for england. Int J Epidemiol. 2012;41(6):1585-1593. doi:10.1093/ije/dyr199

21. Steptoe A, Breeze E, Banks J, Nazroo J. Cohort profile: The English Longitudinal Study of Ageing. Int J Epidemiol. 2013;42(6):1640-1648. doi:10.1093/ije/dys168

22. Guralnik JM, Simonsick EM, Ferrucci L, et al. A Short Physical Performance Battery Assessing Lower Extremity Function: Association With Self-Reported Disability and Prediction of Mortality and Nursing Home Admission. J Gerontol. 1994;49(2):M85-M94. doi:10.1093/geronj/49.2.M85

23. Perracini MR, Mello M, de Oliveira Máximo R, et al. Diagnostic Accuracy of the Short Physical Performance Battery for Detecting Frailty in Older People. Phys Ther. October 2019. doi:10.1093/ptj/pzz154

24. Banks J, Breeze E, Lessof C, Nazroo J. Retirement, Health and Relationships of the Older Population in England: ELSA 2004 (Wave 2):; 2006. 
25. McLean RR, Shardell MD, Alley DE, et al. Criteria for clinically relevant weakness and low lean mass and their longitudinal association with incident mobility impairment and mortality: The Foundation for the National Institutes of Health (FNIH) sarcopenia project. Journals Gerontol - Ser A Biol Sci Med Sci. 2014;69 A(5):576-583. doi:10.1093/gerona/glu012

26. Flegal KM, Carroll MD, Kit BK, Ogden CL. Prevalence of Obesity and Trends in the Distribution of Body Mass Index Among US Adults, 1999-2010. Jama. 2012;307(5):491. doi:10.1001/jama.2012.39

27. Alexandre T da S, Scholes S, Santos JLF, De Oliveira C. Dynapenic Abdominal Obesity as a Risk Factor for Worse Trajectories of ADL Disability Among Older Adults: The ELSA Cohort Study. Journals Gerontol Ser A. 2019;74(7):1112-1118. doi:10.1093/gerona/gly182

28. Alexandre T da S, Scholes S, Ferreira Santos JL, et al. The combination of dynapenia and abdominal obesity as a risk factor for worse trajectories of IADL disability among older adults. Clin Nutr. 2017;in press(6):2045-2053. doi:10.1016/j.clnu.2017.09.018

29. Leeds UK. Joint Health Surveys Unit, National Centre for Social Research and University College London Research Department of Epidemiology and Public Health. The Health Survey for England 2008. doi:10.1016/j.ridd.2011.01.017

30. Steel N, Huppert FA, McWilliams B, et al. Physical and cognitive function. In: Health, Wealth and Lifestyles of the Older Population in England: The 2002 English Longitudinal Study Of Ageing. ; 2003:249.

31. Radloff LS. The CES-D Scale. Appl Psychol Meas. 1977;1(3):385-401. doi:10.1177/014662167700100306

32. de Carvalho DHT, Scholes S, Santos JLF, de Oliveira C, Alexandre T da S. Does Abdominal Obesity Accelerate Muscle Strength Decline in Older Adults? Evidence From the English Longitudinal Study of Ageing. Journals Geronto/ Ser A. 2019;74(7):1105-1111. doi:10.1093/gerona/gly178

33. Vasunilashorn S, Coppin AK, Patel K V., et al. Use of the short physical performance battery score to predict loss of ability to walk 400 meters: Analysis from the InCHIANTI study. Journals Gerontol - Ser A Biol Sci Med Sci. 2009;64(2):223-229. doi:10.1093/gerona/gln022

34. Rabe-Hesketh S, Skrondal A. Multilevel and Longitudinal Modeling Using Stata Vol. II: Categorical Responses, Counts, and Survival.; 2012.

35. Liang K-YY, Zeger SL. Longitudinal Data Analysis Using Generalized Linear Models. Biometrika. 1986;73(1):13-22. doi:10.1093/biomet/73.1.13

36. Zeger SL, Liang K-Y. Longitudinal Data Analysis for Discrete and Continuous Outcomes. Biometrics. 1986;42(1):121. doi:10.2307/2531248

37. Batsis JA, Zbehlik AJ, Pidgeon D, Bartels SJ. Dynapenic obesity and the effect on long-term physical function and quality of life: Data from the osteoarthritis initiative Physical functioning, physical health and activity. BMC Geriatr. 2015;15(1):118. doi:10.1186/s12877-015-0118-9

38. Keevil VL, Luben R, Dalzell N, et al. Cross-sectional associations between different measures of obesity and muscle strength in men and women in a British cohort study. $J$ Nutr Heal Aging. 2015;19(1):3-11. doi:10.1007/s12603-014-0492-6 
39. Akhmedov D, Berdeaux R. The effects of obesity on skeletal muscle regeneration. Front Physiol. 2013;4(December):371. doi:10.3389/fphys.2013.00371

40. Carvalho DHT, Máximo RO, Alexandre TS, Pavarini SCl, Brigola AG. Cognitive impairment as an associated factor of dynapenia in elderly people living in a rural area of Brazil. Alzheimer's Dement. 2017;13(7):P500. doi:10.1016/j.jalz.2017.06.556

\section{Tables}

sble 1. Baseline sociodemographic and behavioral characteristics of older adults according to abdominal obesity and dynapenia status 


\begin{tabular}{|c|c|c|c|c|c|c|c|c|}
\hline & \multicolumn{2}{|c|}{$\mathrm{ND} / \mathrm{NAO}$} & \multicolumn{2}{|c|}{ ND/AO } & \multicolumn{2}{|c|}{ D/NAO } & \multicolumn{2}{|c|}{$\mathrm{D} / \mathrm{AO}$} \\
\hline & Men & Women & Men & Women & Men & Women & Men & Women \\
\hline & $\mathrm{n}=933$ & $\mathrm{n}=882$ & $\mathrm{n}=754$ & $\mathrm{n}=$ & $\mathrm{n}=59$ & $\mathrm{n}=84$ & $\mathrm{n}=35$ & $\mathrm{n}=78$ \\
\hline & & & & 1,056 & & & & \\
\hline \multirow[t]{2}{*}{ Age, years } & $69.5 \pm$ & $69.5 \pm$ & $69.5 \pm$ & $69.5 \pm$ & $79.3 \pm$ & $77.6 \pm$ & $75.9 \pm$ & $74.2 \pm$ \\
\hline & 6.9 & 7.4 & 6.5 & 6.6 & $6.5^{\mathrm{a}, \mathrm{b}}$ & $7.2^{\mathrm{a}, \mathrm{b}}$ & $9.5^{\mathrm{a}, \mathrm{b}}$ & $8.0^{\mathrm{a}, \mathrm{b}, \mathrm{c}}$ \\
\hline Marital status (without conjugal life), & 22.0 & $40.8^{*}$ & 20.6 & $39.7^{*}$ & $40.7^{\mathrm{a}, \mathrm{b}}$ & $67.9^{* a, b}$ & 31.4 & $57.7^{\mathrm{a}, \mathrm{b}}$ \\
\hline \multicolumn{9}{|l|}{$(\%)$} \\
\hline \multicolumn{9}{|l|}{ Total household } \\
\hline $1^{\text {st }}$ quintile (highest), (\%) & 29.6 & 27.2 & $22.9^{\mathrm{a}}$ & $19.0^{\mathrm{a}}$ & 15.3 & $13.1^{\mathrm{a}}$ & $2.9^{\mathrm{a}, \mathrm{b}}$ & $10.3^{\mathrm{a}}$ \\
\hline $2^{\text {nd }}$ quintile, $(\%)$ & 24.0 & 23.4 & 21.4 & 21.3 & 25.4 & 16.7 & 25.7 & 17.9 \\
\hline $3^{\text {rd }}$ quintile, (\%) & 21.5 & 18.8 & 21.5 & 21.8 & 15.3 & 21.4 & 17.1 & 26.9 \\
\hline $4^{\text {th }}$ quintile, (\%) & 14.3 & 16.8 & $19.8^{\mathrm{a}}$ & 20.0 & 23.7 & 22.6 & 28.6 & $30.8^{\mathrm{a}}$ \\
\hline $5^{\text {th }}$ quintile (lowest), (\%) & 9.7 & 12.6 & 13.1 & 16.7 & $20.3^{\mathrm{a}}$ & $26.2^{\mathrm{a}}$ & $22.9^{\mathrm{a}}$ & 14.1 \\
\hline Not reported, (\%) & 0.9 & 1.2 & 1.3 & 1.2 & 0.0 & 0.0 & 2.8 & 0.0 \\
\hline \multicolumn{9}{|l|}{ Level of education } \\
\hline > 13 years, $(\%)$ & 33.4 & $21.8^{*}$ & 27.7 & $16.3^{\mathrm{a}^{*}}$ & 18.6 & $9.5^{\mathrm{a}}$ & $5.7^{\mathrm{a}, \mathrm{b}}$ & $9.0^{\mathrm{a}}$ \\
\hline 12 - 13 years, (\%) & 23.8 & 23.2 & 20.8 & 20.0 & 13.6 & 15.5 & 17.1 & 17.9 \\
\hline $0-11$ years, $(\%)$ & 42.8 & $55.0^{*}$ & $51.5^{\mathrm{a}}$ & $63.7^{\mathrm{a}^{*}}$ & $67.8^{\mathrm{a}}$ & $75.0^{\mathrm{a}}$ & $77.2^{\mathrm{a}, \mathrm{b}}$ & $73.1^{\mathrm{a}}$ \\
\hline \multicolumn{9}{|l|}{ Smoking } \\
\hline Non-smoker, (\%) & 30.6 & $47.3^{*}$ & $24.5^{\mathrm{a}}$ & $44.6^{*}$ & $15.2^{\mathrm{a}}$ & $48.8^{*}$ & 17.1 & 42.3 \\
\hline Ex-smoker, (\%) & 56.4 & $41.0^{*}$ & 64.7 & $45.2^{*}$ & $67.8^{\mathrm{a}}$ & $41.7^{*}$ & 74.3 & 48.7 \\
\hline Smoker, (\%) & 13.0 & 11.7 & 10.8 & 10.2 & 17.0 & 9.5 & 8.6 & 9.0 \\
\hline \multicolumn{9}{|l|}{ Alcohol intake } \\
\hline Non-drinker or rare drinker, (\%) & 10.1 & $20.0^{*}$ & 11.5 & $23.9^{*}$ & 8.5 & 27.4 & $5.7^{\mathrm{b}}$ & $30.8^{*}$ \\
\hline Frequent drinker, (\%) & 39.6 & 43.4 & 42.2 & 43.8 & 44.1 & 40.5 & 45.7 & 39.7 \\
\hline Daily drinker, (\%) & 42.3 & $30.5^{*}$ & 38.3 & $23.6^{*}$ & $23.7^{\mathrm{a}}$ & 20.2 & 22.9 & 21.8 \\
\hline Did not answer, (\%) & 8.0 & 6.1 & 8.0 & 8.7 & $23.7^{\mathrm{a}, \mathrm{b}}$ & 11.9 & $25.7^{\mathrm{a}, \mathrm{b}}$ & 7.7 \\
\hline Sedentary lifestyle, (\%) & 2.5 & 1.7 & 2.1 & 2.9 & 5.1 & $7.1^{\mathrm{a}}$ & 2.9 & $7.7^{\mathrm{a}}$ \\
\hline
\end{tabular}

ND/NAO: Non-Dynapenic/Non-Abdominal Obese; ND/AO: Non-Dynapenic/Abdominal Obese; D/NAO: Dynapenic/Non-Abdominal Obese; D/AO: Dynapenic/Abdominal Obese. Data expressed as percentage, mean and standard deviation (SD). * Significant sex difference in each group. ${ }^{*}$ 
Significantly different from ND/NAO for men and women; ${ }^{b}$ Significantly different from ND/AO for men and women; ${ }^{c}$ Significantly different from D/NAO for men and women. Statistical significance $\mathrm{p}<0.05$.

Table 2. Baseline clinical characteristics of older adults according to abdominal obesity and dynapenia status

\begin{tabular}{|c|c|c|c|c|c|c|c|c|}
\hline & \multicolumn{2}{|c|}{ ND/NAO } & \multicolumn{2}{|c|}{$\mathrm{ND} / \mathrm{AO}$} & \multicolumn{2}{|c|}{ D/NAO } & \multicolumn{2}{|c|}{$\mathrm{D} / \mathrm{AO}$} \\
\hline & Men & Women & Men & Women & Men & Women & Men & Women \\
\hline & $\mathrm{n}=933$ & $\mathrm{n}=882$ & $\mathrm{n}=754$ & $\mathrm{n}=1,056$ & $\mathrm{n}=59$ & $\mathrm{n}=84$ & $\mathrm{n}=35$ & $\mathrm{n}=78$ \\
\hline Arterial Hypertension (sim), & 36.3 & 38.0 & $52.7^{\mathrm{a}}$ & $55.2^{\mathrm{a}}$ & 42.4 & 46.4 & 54.3 & $64.1^{\mathrm{a}}$ \\
\hline \multicolumn{9}{|l|}{$(\%)$} \\
\hline Diabetes (sim), (\%) & 7.9 & $2.3^{*}$ & 11.9 & $9.9^{\mathrm{a}}$ & 11.9 & 4.8 & $25.7^{\mathrm{a}}$ & $14.1^{\mathrm{a}}$ \\
\hline Cancer (yes), (\%) & 7.1 & 9.8 & 9.8 & 9.6 & 8.5 & 13.1 & 8.6 & 9.0 \\
\hline Lung disease (yes), (\%) & 14.7 & 16.8 & 16.7 & 20.6 & 13.6 & 16.7 & 28.6 & 24.4 \\
\hline Heart disease (yes), (\%) & 25.8 & 20.5 & 26.1 & 20.9 & 32.2 & 28.6 & 34.3 & 28.2 \\
\hline Stroke (yes), (\%) & 4.9 & 3.6 & 4.4 & 3.2 & 5.1 & $9.5^{\mathrm{b}}$ & 2.9 & 5.1 \\
\hline Joint disease, (\%) & 22.7 & $36.6^{*}$ & $32.2^{\mathrm{a}}$ & $45.6^{a^{*}}$ & $44.1^{\mathrm{a}}$ & $71.4^{* a, b}$ & $51.4^{\mathrm{a}}$ & $75.6^{\mathrm{a}, \mathrm{b}}$ \\
\hline Osteoporosis, (\%) & 1.2 & $13.0^{*}$ & 1.7 & $9.8^{*}$ & 3.4 & 15.5 & 8.6 & 15.4 \\
\hline Depressive symptoms, (\%) & 6.8 & $11.1^{*}$ & 7.8 & $15.4^{* a}$ & 10.2 & $23.8^{\mathrm{a}}$ & 11.4 & 16.7 \\
\hline \multirow[t]{2}{*}{ Memory Score, points } & $9.5 \pm 3.2$ & $10.3 \pm$ & $9.3 \pm 3.2$ & $10.0 \pm 3.3^{*}$ & $6.8 \pm 3.4^{\mathrm{a}, \mathrm{b}}$ & $8.0 \pm 4.1^{\mathrm{a}, \mathrm{b}}$ & $8.3 \pm 3.7$ & $8.7 \pm 3.7^{a, b}$ \\
\hline & & $3.5^{*}$ & & & & & & \\
\hline \multirow[t]{2}{*}{ Grip strength, kg } & $39.6 \pm$ & $24.4 \pm$ & $40.6 \pm 7.5^{\mathrm{a}}$ & $24.7 \pm 5.0^{*}$ & $21.0 \pm$ & $12.7 \pm$ & $22.5 \pm 2.9^{a, b}$ & $13.1 \pm$ \\
\hline & 7.4 & $4.9^{*}$ & & & $4.8^{\mathrm{a}, \mathrm{b}}$ & $2.4^{* a, b}$ & & $2.6^{* a, b}$ \\
\hline \multirow[t]{2}{*}{ Waist circumference, $\mathrm{cm}$} & $93.7 \pm$ & $80.2 \pm$ & $110.3 \pm$ & $98.5 \pm$ & $92.5 \pm 7.0^{\mathrm{b}}$ & $79.5 \pm 6.1^{* \mathrm{~b}}$ & $109.6 \pm$ & $97.9 \pm$ \\
\hline & 6.2 & $5.6^{*}$ & $7.0^{\mathrm{a}}$ & $8.3^{* a}$ & & & $5.6^{\mathrm{a}, \mathrm{c}}$ & $8.2^{* a, c}$ \\
\hline
\end{tabular}

ND/NAO: Non-Dynapenic/Non-Abdominal Obese; ND/AO: Non-Dynapenic/Abdominal Obese; D/NAO: Dynapenic/Non-Abdominal Obese; D/AO: Dynapenic/Abdominal Obese. Data expressed as percentage, mean and standard deviation (SD). * Significant sex difference in each group. a Significantly different from ND/NAO for men and women; ${ }^{b}$ Significantly different from ND/AO for men and women; ${ }^{c}$ Significantly different from D/NAO for men and women. Statistical significance $\mathrm{p}<0.05$.

Table 3. GLMM estimated for SPPB scores as function of abdominal obesity and dynapenia status over eight-years follow-up 


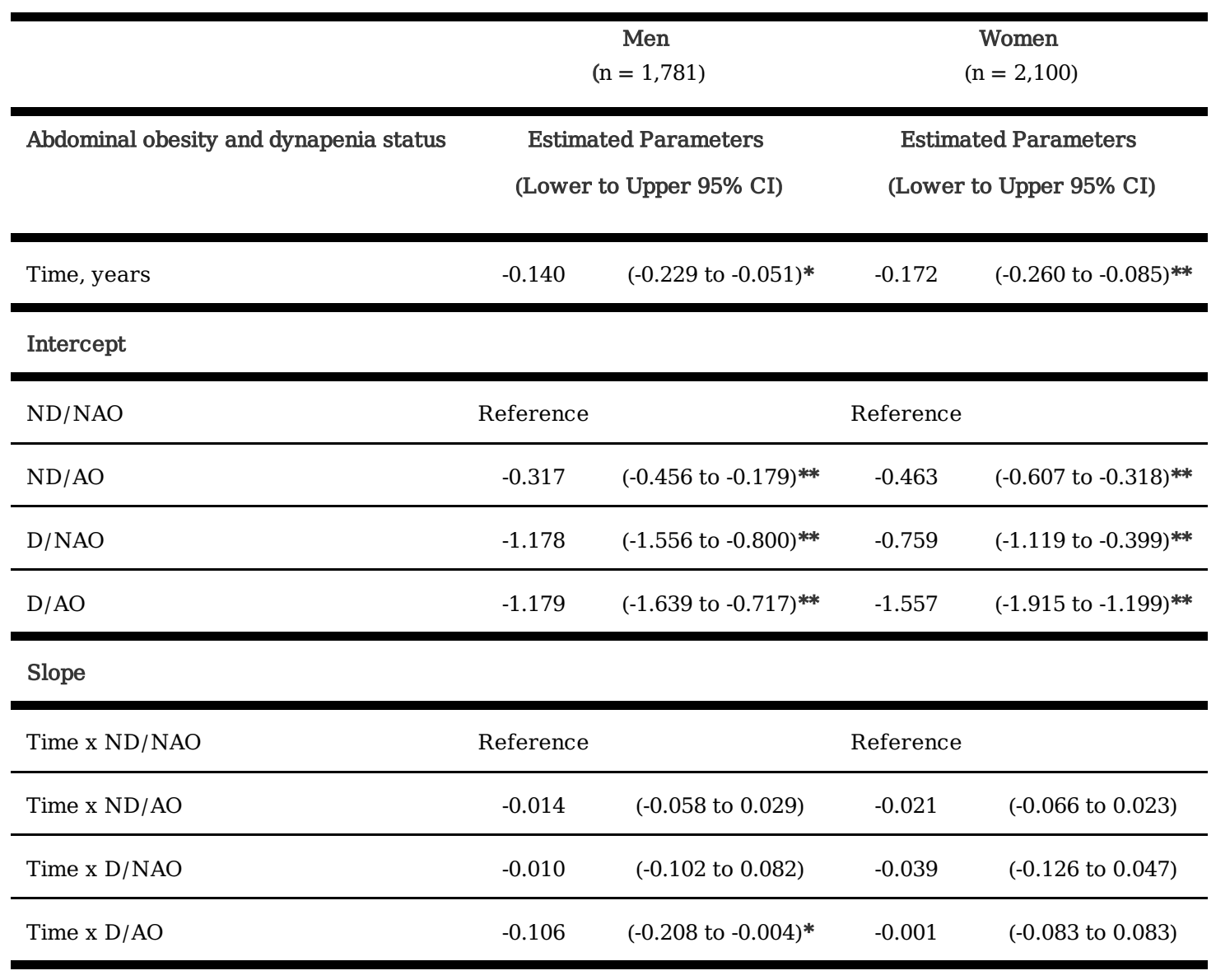

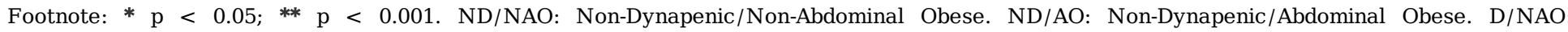

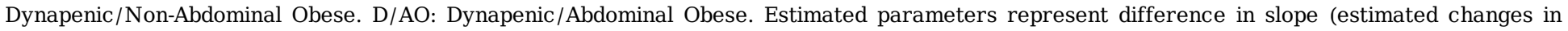

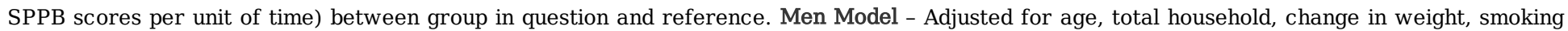

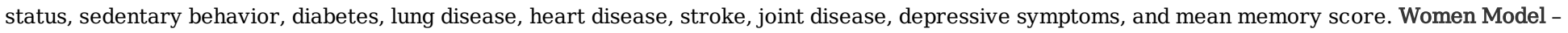

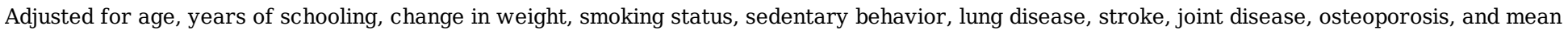
memory score. GLMM=Generalized linear mixed model.

\section{Figures}




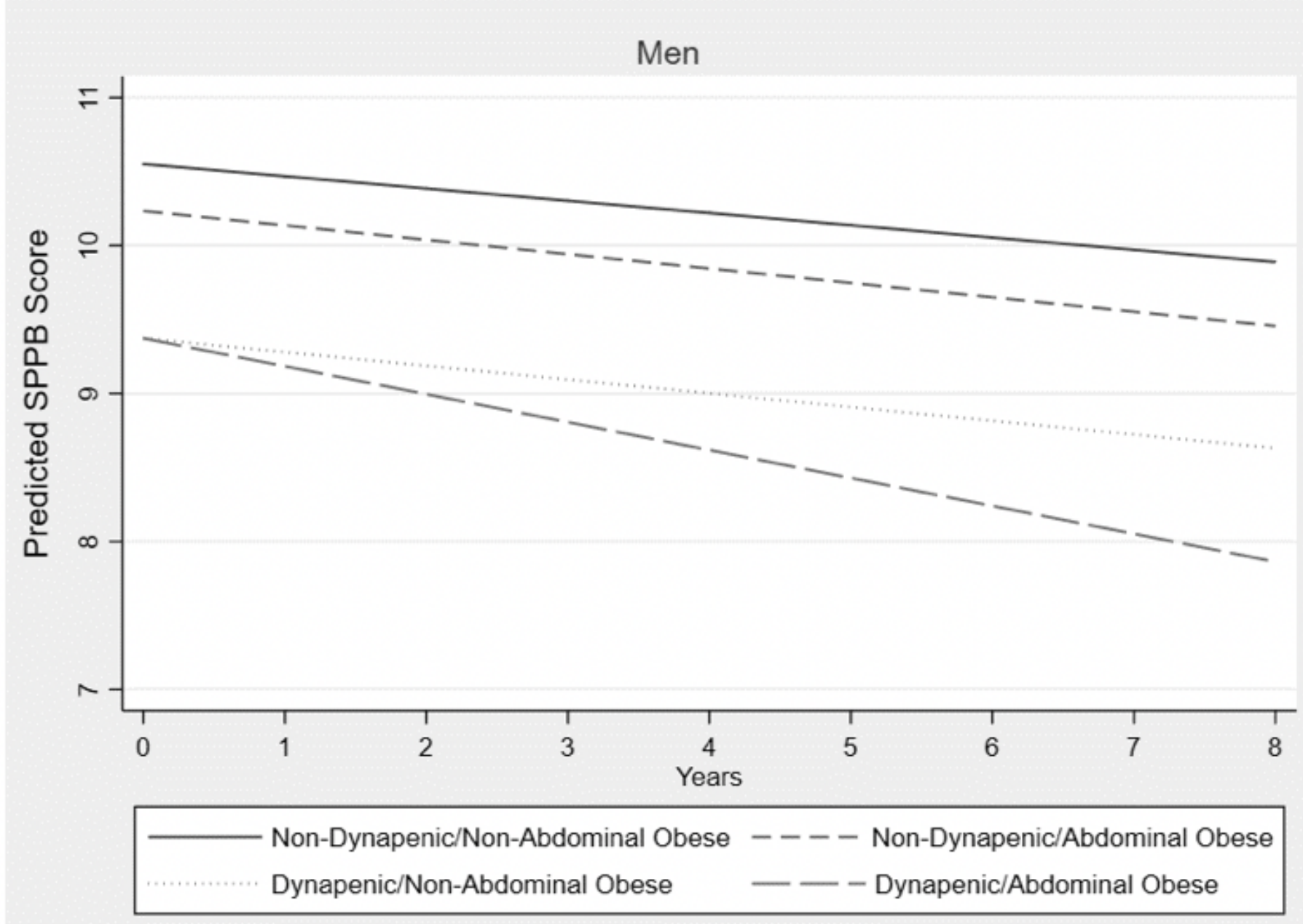

\section{Figure 1}

Trajectories of performance on SPPB according to abdominal obesity and dynapenia status - ELSA Study 2004-2012. Predictions for 60 years of age, men, total household $=1$ st quintile, non-smokers, active, without diabetes, without lung disease, without heart disease, without stroke, without joint disease, CESD $<4$ points, mean Memory Score $=20$ and stable weight. 


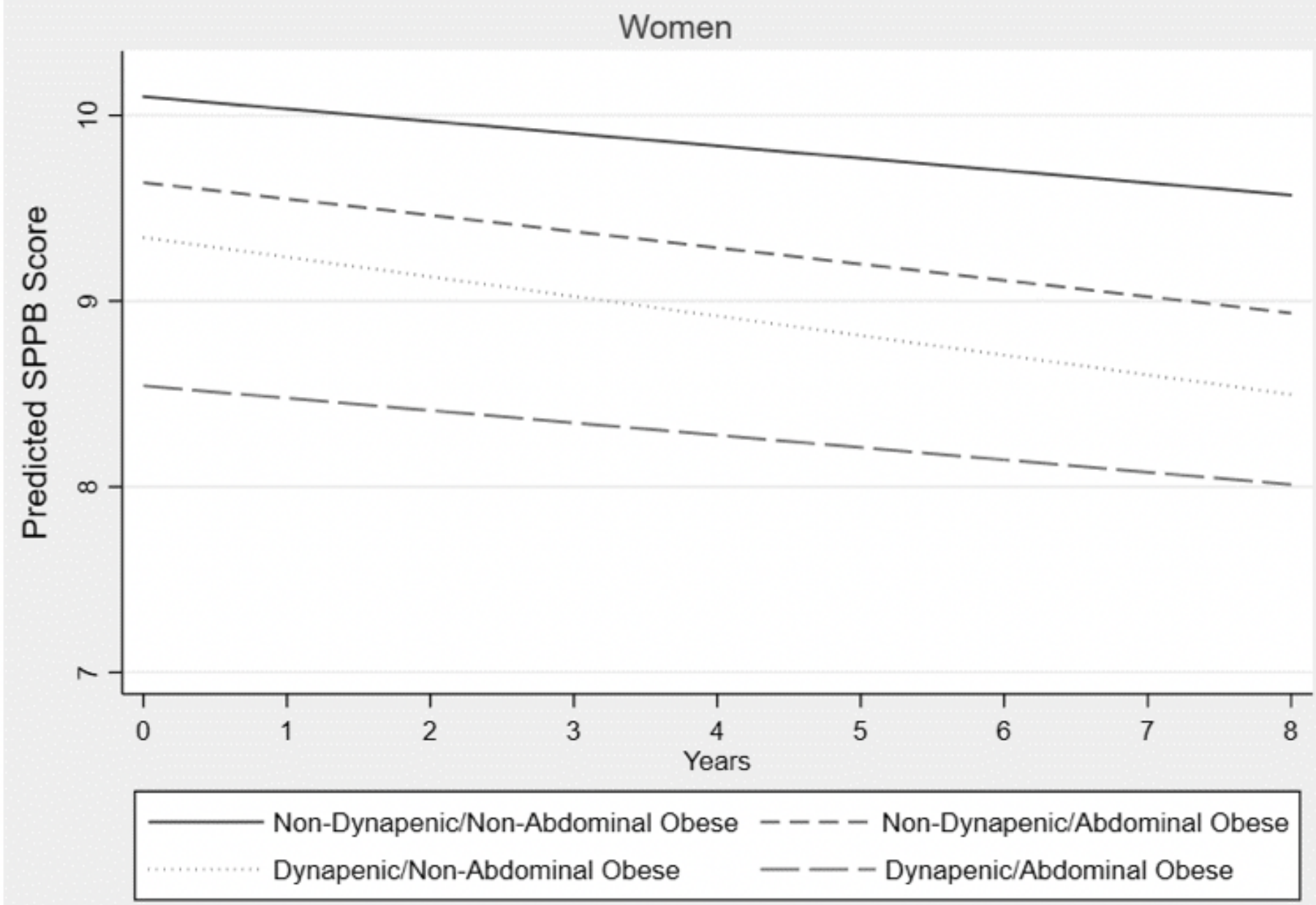

\section{Figure 3}

Trajectories of performance on SPPB according to abdominal obesity and dynapenia status - ELSA Study 2004-2012. Predictions for 60 years of age, women, level of education $>13$ years, non-smokers, active, without diabetes, without lung disease, without stroke, without joint disease, without osteoporosis, mean Memory Score $=20$ and stable weight.

\section{Supplementary Files}

This is a list of supplementary files associated with this preprint. Click to download.

- Supplementary1TablelJBNP.docx

- Supplementary2TableIJBNP.docx

- Supplementary1TablelJBNP.docx

- Supplementary2TableIJBNP.docx 